\title{
Moves app: a digital diary to track physical activity and location
}

\author{
Kelly R Evenson, ${ }^{1,2}$ Robert D Furberg ${ }^{2}$
}

'Department of Epidemiology, Gillings School of Global Public Health, University of North Carolina-Chapel Hill, Chapel Hill, North Carolina, USA ${ }^{2}$ RTI International, Research Triangle Park, North Carolina, USA

\section{Correspondence to}

Dr Kelly R Evenson,

Department of Epidemiology, University of NC, Gillings

School of Global Public Health, 137 East Franklin Street, Suite 306, Chapel Hill, North Carolina 27514, USA; kelly_evenson@unc.edu

Accepted 23 May 2016
To cite: Evenson KR, Furberg RD. Br I Sports Med Published Online First: [please include Day Month Year] doi:10.1136/bjsports2016-096103

\section{CATEGORY Fitness.}

PLATFORM iOS 9.0 or later, Android 4.0.3 or later.

COST Free.

\section{ABOUT THE APP}

The Moves app (moves-app.com) was launched in January 2013. The app uses global positioning system (GPS) technology, Wi-Fi and cell towers along with samples of the smartphone's accelerometer data to record location, physical activity (eg, walking, bicycling and running) and transit throughout the day. ${ }^{1}$ In the account settings, users can provide their email, gender, weight, height and birth year. From these assessments, the app provides an estimate of physical activity duration, distance, steps and calories expended. The Moves app graphs a storyline of the day by accounting for time spent in travel and visiting destinations. Daily routes are automatically mapped in different colours based on the type of transport (eg, walking, bicycling, and public transit). The company uses 'commercially reasonable physical and technical safeguards to secure' user's data, such as using encryption using secure sockets layer (SSL), but does not guarantee the security of the data, which may be compromised by hardware or software failure or unauthorised entry or use. ${ }^{1}$

For research purposes, studies have generally required participants to wear both a GPS and an accelerometer in precise locations (such as on the hip) in order to combine location and physical activity information. $^{2}$ The Moves app provides both GPS and accelerometer data for intraday, day and week-long epochs or lengths of time. Variables include time-stamped geolocation coordinates, physical activity duration, distance, steps and calories expended, and are available to download in a variety of file formats including CSV, KML, JSON and GPX (https://dev.moves-app.com/). If the variables are shown to be both reliable and valid, and if security of the data can be ensured, the Moves app may provide researchers an alternative approach with lower cost and less participant burden.

\section{USE IN CLINICAL PRACTICE}

The Moves app can support patients to be physically active. The behaviour change techniques offered in this app include helping users create physical activity-related goals, providing feedback and self-monitoring of behaviour. ${ }^{3}$ Moreover, Moves currently integrates with over 40 'connected

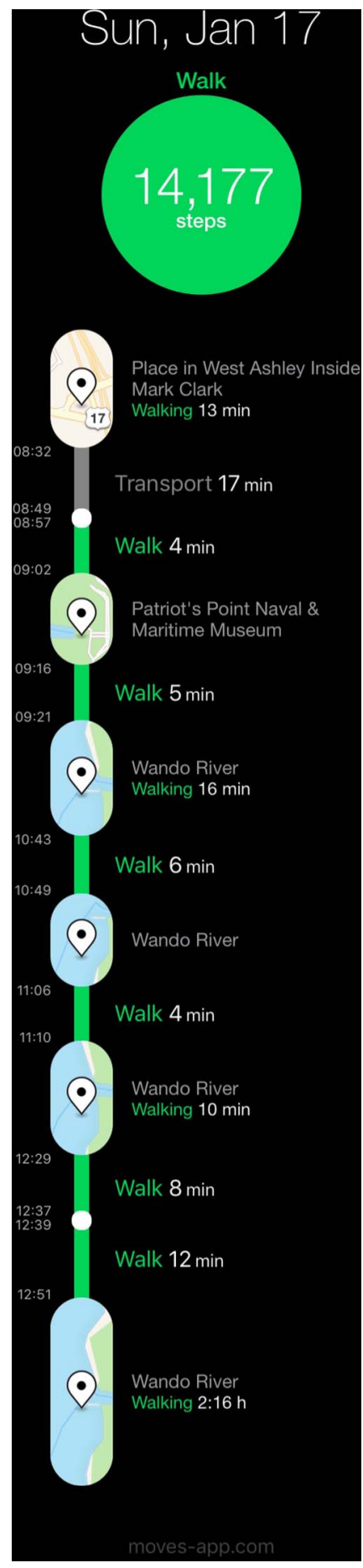

apps' (https://apps.moves-app.com/), including other health-related motivating tools, mapping utilities and data visualisation applications. A robust Application Programming Interface library also enables third-party developers to build applications that use data from Moves. This pairing could 
provide the clinician with a variety of daily health-oriented data on their patients to integrate with the physical activity data provided by the app.

\section{PROS}

- Tracks daily physical activity in a user-friendly format that accounts for location;

- Provides a calorie counter for energy expended;

- Easy to use, running continuously on mobile devices for passive data collection;

- Offered in Chinese (simplified and traditional), English, French, German, Italian, Japanese, Korean, Portuguese and Spanish with other languages being added;

- Can connect to other apps and provides easy to use tools for exporting data;

- Allows users an option to download a copy of their data;

- Provides a way to explore whether purchasing an activity tracker would be useful. ${ }^{4}$

\section{CONS}

- Does not provide a calorie counter for energy intake;

- High battery usage due to GPS tracking; the company recommends nightly phone charging and users may also want to use the battery optimisation option, which will reduce battery usage when the phone is not moving;
- Unknown validity of the measures it provides (eg, physical activity duration, distance, steps, calories expended);

- Although the data are encrypted using SSL, the security of the data is not guaranteed. ${ }^{1}$

Contributors KRE drafted the article and RDF reviewed the article several times, and made substantial contributions. Both authors approved the final version submitted to the journal.

Funding RTI International through the RTI University Scholars Program and iSHARE.

Disclaimer The content is solely the responsibility of the authors and does not necessarily represent the official views of RTI International.

Competing interests None declared.

Provenance and peer review Not commissioned; externally peer reviewed.

\section{REFERENCES}

1 Moves app: Privacy policy. Last updated 5 May 2014 (accessed 30 March 2016). https://moves-app.com/privacy

2 Kerr J, Duncan S, Schipperijn J. Using global positioning systems in health research: a practical approach to data collection and processing. Am J Prev Med 2011:41:532-40

3 Middelweerd A, Mollee JS, van der Wal CN, et al. Apps to promote physical activity among adults: a review and content analysis. Int I Behav Nutr Phys Act 2014;11:97.

4 Moves (for iPhone). PC Reviews. Published February 252014 (accessed 30 Mar 2016). http://www.pcmag.com/article2/0,2817,2454045,00.asp 